\title{
LOCAL AND GLOBAL LIFTINGS OF ANALYTIC FAMILIES OF IDEMPOTENTS IN BANACH ALGEBRAS
}

\author{
DEDICATED TO THE 100TH ANNIVERSARY \\ OF PROFESSOR BÉLA SZŐKEFALVI-NAGY
}

\author{
Bernard Aupetit, Endre Makai, Jr., \\ Mostafa Mbekhta, and Jaroslav Zemánek
}

\begin{abstract}
Generalizing results of our earlier paper, we investigate the following question. Let $\pi(\lambda): A \rightarrow B$ be an analytic family of surjective homomorphisms between two Banach algebras, and $q(\lambda)$ an analytic family of idempotents in $B$. We want to find an analytic family $p(\lambda)$ of idempotents in $A$, lifting $q(\lambda)$, i.e., such that $\pi(\lambda) p(\lambda)=q(\lambda)$, under hypotheses of the type that the elements of $\operatorname{Ker} \pi(\lambda)$ have small spectra. For spectra which do not disconnect $\mathbb{C}$ we obtain a local lifting theorem. For real analytic families of surjective ${ }^{*}$-homomorphisms (for continuous involutions) and self-adjoint idempotents we obtain a local lifting theorem, for totally disconnected spectra. We obtain a global lifting theorem if the spectra of the elements in $\operatorname{Ker} \pi(\lambda)$ are $\{0\}$, both in the analytic case, and, for ${ }^{*}$-algebras (with continuous involutions) and self-adjoint idempotents, in the real analytic case. Here even an at most countably infinite set of mutually orthogonal analytic families of idempotents can be lifted to mutually orthogonal analytic families of idempotents. In the proofs, spectral theory is combined with complex analysis and general topology, and even a connection with potential theory is mentioned.
\end{abstract}

\section{Notations}

For a Banach algebra $A$, let

$$
E(A):=\left\{p \in A: p^{2}=p\right\}
$$

be the set of idempotents. If there is an involution * on $A$, let

$$
S(A):=\left\{p \in A: p^{2}=p=p^{*}\right\}
$$

be the set of self-adjoint idempotents.

For a Banach space $X$, let $\mathcal{B}(X)$ or $\mathcal{K}(X)$ denote the Banach algebras of all bounded or all compact linear operators on $X$, respectively. (For two Banach

2010 Mathematics Subject Classification. Primary 46H05; Secondary 46T25, 47B48, 47L10.

Key words and phrases. Banach algebras, homomorphisms, idempotents, analytic families, liftings.

Research (partially) supported by Hungarian National Foundation for Scientific Research, grant numbers K68398, K75016, K81146, and the European Commission Project TODEQ (MTKD-CT2005-030042). 
spaces $X, Y$, let $\mathcal{B}(X, Y)$ denote the Banach space of all bounded linear operators $X \rightarrow Y$.) The quotient $\mathcal{C}(X):=\mathcal{B}(X) / \mathcal{K}(X)$ is called the Calkin algebra (as it was first studied in $[\mathrm{C}]$ for the Hilbert space case). Let

$$
\pi: \mathcal{B}(X) \rightarrow \mathcal{C}(X)
$$

be the canonical map. More generally, we will consider situations where $\pi: A \rightarrow B$ is a surjective map between Banach algebras.

In this paper, we will study the relationship between $E(A)$ and $E(A / I)$, where $I$ is a closed two-sided ideal in $A$. Observe that

$$
\pi E(A) \subset E(A / I)
$$

where $\pi: A \rightarrow A / I$ is the canonical map. Thus, the lifting problem consists in establishing the converse inclusion. See Gramsch [Gr] for a case, when the converse inclusion does not hold (cf. also [AMMZ, p. 26]).

In this paper, the linear structures are considered over the complex scalars $\mathbb{C}$, the algebras are assumed to be unital (unless explicitly stated contrarily), and all Banach algebra homomorphisms are assumed to be continuous, and preserving 1. We denote by $\sigma(x)$ the spectrum of an element $x$ of a Banach algebra. When considering spectra of elements of various kernels of mappings $A \rightarrow B$, which kernels are therefore subsets of the Banach algebra $A$, the spectra are considered with respect to $A$.

\section{LifTing OF IDEMPOTENTS AND LOCAL LIFTING OF ANALYTIC FAMILIES OF IDEMPOTENTS}

The lifting of a single self-adjoint idempotent was first studied for the case $A=$ $\mathcal{B}(H)$ and $I=\mathcal{K}(H)$, where $H$ is a Hilbert space, with the result that $\pi S(\mathcal{B}(H))=$ $S(\mathcal{C}(H)$ ), see [C, Theorem 2.4] and [dlH, Proposition 7]. For a general Banach algebra $A$ and $I=\operatorname{rad} A$, the lifting property $\pi E(A)=E(A / \operatorname{rad} A)$ can be found in [Ri, p. 58, Theorem 2.3.9] and [Ka, p. 125]. The formula $\pi E(\mathcal{B}(H))=E(\mathcal{C}(H))$ was obtained in [La, Theorem 2]. Labrousse actually proved that each analytic family of idempotents of $E(\mathcal{C}(H)$ ) can be locally lifted to an analytic family of idempotents in $E(\mathcal{B}(H))$. That is, for each analytic map $q: U \rightarrow E(\mathcal{C}(H))$, where $U$ is an open set in $\mathbb{C}$, any point of $U$ has an open neighbourhood $V \subset U$, such that there exists an analytic map $p: V \rightarrow E(\mathcal{B}(H))$ with $\pi p(\lambda)=q(\lambda)$ for each $\lambda \in V$, with $\pi: \mathcal{B}(H) \rightarrow \mathcal{C}(H)$ being the canonical map. Recall that every compact operator on a Banach space has a spectrum that is finite, or is a sequence converging to 0 , together with 0 ([DS, p. 579, Theorem VII. 4.5]). Such a compact set is totally disconnected (i.e., it contains no connected subset consisting of more than one points), which implies, due to compactness, that its complement in $\mathbb{C}$ is connected $([\mathrm{Ku}, \mathrm{p} .466$, Section 59, II, Theorem 1, and p. 188, Section 47, VIII, Theorem 1]). (Cf. also the remarks before the proof of our Theorem 2.) This raises the question, what can be said in general about the equality $\pi E(A)=E(A / I)$, if we make restrictions on the spectra of the elements in $I=\pi^{-1}(0)=\operatorname{Ker} \pi$.

In $[\mathrm{AMMZ}]$ there were proved four theorems of this type. 
Theorem A. ([AMMZ, Theorem 3.1 and Corollary 3.3]) Let $\pi: A \rightarrow B$ be a surjective homomorphism between Banach algebras. Suppose that the spectrum of each element of $\operatorname{Ker} \pi$ is totally disconnected. Let $U \subset \mathbb{C}$ be open, and let $q: U \rightarrow$ $E(B)$ be an analytic map. Then any point of $U$ has an open neighbourhood $V \subset U$, such that there exists an analytic map $p: V \rightarrow E(A)$ with $\pi p(\lambda)=q(\lambda)$ for each $\lambda \in V$. In particular, for $q$ constant, we obtain $\pi E(A)=E(B)$.

Theorem B. ([AMMZ, Corollary 3.3]) Assume the hypotheses of Theorem A, and let $A$ and $B$ possess continuous involutions, and let $\pi$ be $a^{*}$-homomorphism. Then $\pi S(A)=S(B)$.

Theorem C. ([AMMZ, p. 26, paragraph following the proof of Corollary 3.3]) Let $\pi: A \rightarrow B$ be a surjective homomorphism of Banach algebras. Suppose that the spectrum of each element of $\operatorname{Ker} \pi$ does not disconnect $\mathbb{C}$. Then $\pi E(A)=E(B)$.

Theorem D. ([AMMZ, Corollary 3.2]) For the canonical map $\pi: A \rightarrow A / \operatorname{rad} A$ we may choose $V=U$ in Theorem A, i.e., there is a global lifting of $q(\lambda)$.

We cite below a restriction of a theorem from [Ka, pp. 125-126] to Banach algebras. Before this we recall that two idempotents $e$ and $f$ of a Banach algebra are orthogonal, if ef $=f e=0$.

Theorem E. ([Ka, pp. 125-126]) If $A$ is a Banach algebra and $q_{1}, q_{2}, \ldots$ is a finite or countably infinite set of orthogonal idempotents in $A / \mathrm{rad} A$, then there are orthogonal idempotents $p_{1}, p_{2}, \ldots$ in $A$ (indexed by the same index set as the $q_{i}$ 's), such that $\pi\left(p_{i}\right)=q_{i}$, for all $i$ 's, where $\pi$ is the canonical map $A \rightarrow A / \operatorname{rad} A$.

In this paper we generalize the above theorems.

For a more comprehensive literature on the structure of the set of idempotents, we refer to [AMMZ, §2, Historical background].

\section{LOCAL AND GLOBAL LIFTINGS OF ANALYTIC FAMILIES OF}

IDEMPOTENTS, FOR ANALYTIC FAMILIES OF SURJECTIVE HOMOMORPHISMS

We give a common generalization of Theorems A and C.

Theorem 1. Let $U$ be an open subset of $\mathbb{C}$, containing 0 . Let $A$ and $B$ be Banach algebras, and let $\pi: U \rightarrow \mathcal{B}(A, B)$ be an analytic map, whose values are homomorphisms $A \rightarrow B$, such that $\pi(0)$ is surjective. Suppose that the spectrum of each element of $\operatorname{Ker} \pi(0)$ does not disconnect $\mathbb{C}$. Let $q: U \rightarrow E(B)$ be an analytic map. Then there exist an open set $V \subset \mathbb{C}$, such that $0 \in V \subset U$, and an analytic map $p: V \rightarrow E(A)$, such that $\pi(\lambda) p(\lambda)=q(\lambda)$ for each $\lambda \in V$.

We prove a generalization of Theorem B. By a real analytic map from an open subset $G$ of $\mathbb{R}$ to a Banach space, we mean a map $f$ that for each $x_{0} \in G$ has locally a power series expansion $f(x)=\sum_{0}^{\infty} a_{n}\left(x-x_{0}\right)^{n}$. In the real analytic case, we need a stronger spectral assumption (total disconnectedness), again, for one of the kernels (namely, at 0 ), merely. Then we have a variant of Theorem 1, for Banach algebras with continuous involutions.

Theorem 2. Let $G$ be an open subset of $\mathbb{R}$, containing 0 . Let $A$ and $B$ be Banach 
algebras with continuous involutions, and let $\pi: G \rightarrow \mathcal{B}(A, B)$ be a real analytic map, whose values are ${ }^{*}$-homomorphisms $A \rightarrow B$, such that $\pi(0)$ is surjective. Suppose that the spectrum of each element of $\operatorname{Ker} \pi(0)$ is totally disconnected. Let $q: G \rightarrow S(B)$ be a real analytic map. Then there exist an open set $H \subset \mathbb{R}$, such that $0 \in H \subset G$, and a real analytic map $p: H \rightarrow S(A)$, such that $\pi(\lambda) p(\lambda)=q(\lambda)$ for each $\lambda \in H$.

We give a common generalization of Theorems $\mathrm{D}$ and E. Recall that for the canonical map $\pi: A \rightarrow A / \operatorname{rad} A$ we have $\sigma(a)=\sigma(\pi(a))$ for each $a \in A$, cf. [A79, p. 2, Lemme 1.1.2], or [A91, p. 35, Theorem 3.1.5]. Here the strongest spectral assumption is imposed on all the kernels in question.

Theorem 3. Let $U$ be an open subset of $\mathbb{C}$. Let $A$ and $B$ be Banach algebras, and let $\pi: U \rightarrow \mathcal{B}(A, B)$ be an analytic map, whose values are surjective homomorphisms $A \rightarrow B$. Suppose that the spectrum of each element of $\operatorname{Ker} \pi(\lambda)$, for each $\lambda \in U$, is $\{0\}$. Let $q_{1}, q_{2}, \cdots: U \rightarrow E(B)$ be finitely or countably infinitely many analytic maps, where for each $i \neq j$ and each $\lambda \in U$ we have that $q_{i}(\lambda)$ and $q_{j}(\lambda)$ are orthogonal. Then there exist analytic maps $p_{1}, p_{2}, \cdots: U \rightarrow E(A)$ (indexed by the same index set as the $q_{i}$ 's), where for each $i \neq j$ and each $\lambda \in U$ we have that $p_{i}(\lambda)$ and $p_{j}(\lambda)$ are orthogonal, and such that $\pi(\lambda) p_{i}(\lambda)=q_{i}(\lambda)$ holds for each index $i$ and each $\lambda \in U$.

We give a variant of Theorem 3 for Banach algebras with continuous involutions.

Theorem 4. Let $G$ be an open subset of $\mathbb{R}$. Let $A$ and $B$ be Banach algebras with continuous involutions, and let $\pi: G \rightarrow \mathcal{B}(A, B)$ be a real analytic map, whose values are surjective ${ }^{*}$-homomorphisms $A \rightarrow B$. Suppose that the spectrum of each element of $\operatorname{Ker} \pi(\lambda)$, for each $\lambda \in G$, is $\{0\}$. Let $q_{1}, q_{2}, \cdots: G \rightarrow S(B)$ be finitely or countably infinitely many real analytic maps, where for each $i \neq j$ and each $\lambda \in G$ we have that $q_{i}(\lambda)$ and $q_{j}(\lambda)$ are orthogonal. Then there exist real analytic maps $p_{1}, p_{2}, \cdots: G \rightarrow S(A)$ (indexed by the same index set as the $q_{i}$ 's), where for each $i \neq j$ and each $\lambda \in G$ we have that $p_{i}(\lambda)$ and $p_{j}(\lambda)$ are orthogonal, and such that $\pi(\lambda) p_{i}(\lambda)=q_{i}(\lambda)$ holds for each index $i$ and each $\lambda \in G$.

In the next two theorems, the strongest spectral assumption is imposed only on one of the kernels (namely, at 0). But then we are able to lift only a finite set of analytic families of orthogonal idempotents, and only locally.

Theorem 5. Let $U$ be an open subset of $\mathbb{C}$, containing 0 . Let $A$ and $B$ be $B a$ nach algebras, and let $\pi: U \rightarrow \mathcal{B}(A, B)$ be an analytic map, whose values are homomorphisms $A \rightarrow B$, such that $\pi(0)$ is surjective. Suppose that the spectrum of each element of $\operatorname{Ker} \pi(0)$ is $\{0\}$. Let $q_{1}, \ldots, q_{n}: U \rightarrow E(B)$ be finitely many analytic maps, where for each $i \neq j$ and each $\lambda \in U$ we have that $q_{i}(\lambda)$ and $q_{j}(\lambda)$ are orthogonal. Then there exist an open set $V \subset \mathbb{C}$, such that $0 \in V \subset U$, and analytic maps $p_{1}, \ldots, p_{n}: V \rightarrow E(A)$, where for each $i \neq j$ and each $\lambda \in V$ we have that $p_{i}(\lambda)$ and $p_{j}(\lambda)$ are orthogonal, and such that $\pi(\lambda) p_{i}(\lambda)=q_{i}(\lambda)$ holds for each index $i \in\{1, \ldots, n\}$ and each $\lambda \in V$.

The following Theorem 6 is a variant of Theorem 5, for Banach algebras with continuous involutions. 
Theorem 6. Let $G$ be an open subset of $\mathbb{R}$, containing 0 . Let $A$ and $B$ be Banach algebras with continuous involutions, and let $\pi: G \rightarrow \mathcal{B}(A, B)$ be a real analytic map, whose values are ${ }^{*}$-homomorphisms $A \rightarrow B$, such that $\pi(0)$ is surjective. Suppose that the spectrum of each element of $\operatorname{Ker} \pi(0)$ is $\{0\}$. Let $q_{1}, \ldots, q_{n}: G \rightarrow$ $S(B)$ be finitely many real analytic maps, where for each $i \neq j$ and each $\lambda \in G$ we have that $q_{i}(\lambda)$ and $q_{j}(\lambda)$ are orthogonal. Then there exist an open set $H \subset \mathbb{R}$, such that $0 \in H \subset G$, and real analytic maps $p_{1}, \ldots, p_{n}: H \rightarrow S(A)$, where for each $i \neq j$ and each $\lambda \in H$ we have that $p_{i}(\lambda)$ and $p_{j}(\lambda)$ are orthogonal, and such that $\pi(\lambda) p_{i}(\lambda)=q_{i}(\lambda)$ holds for each index $i \in\{1, \ldots, n\}$ and each $\lambda \in H$.

Remark 1. In fact our theorems hold in greater generality, as the proofs given show this. Theorems 1 and 5 hold for $0 \in U \subset \mathbb{C}^{n}$ open. Theorems 2 and 6 hold for $0 \in G \subset \mathbb{R}^{n}$ open. Theorem 3 holds if we replace $U$ by a Stein manifold. Theorem 4 holds for $G \subset \mathbb{R}^{n}$ open, provided each connected component of $U$ has a neighbourhood base consisting of domains of holomorphy, when we consider $\mathbb{R}^{n}$ as embedded in $\mathbb{C}^{n}$ in the canonical way.

Remark 2. Another way of strengthening our theorems, for Theorems 3 and 4 , is the following. Retaining the hypothesis $U \subset \mathbb{C}$ or $G \subset \mathbb{R}$ open, the spectral hypothesis, about the spectra of elements of $\operatorname{Ker} \pi(\lambda)$, is not necessary to be postulated for all $\lambda$ in $U$, or in $G$, respectively. For Theorems 3 and 4 it is sufficient to postulate it for some subsets of each connected component of $U$, or $G$, respectively, which have positive outer capacity (like, e.g., non-degenerate straight line segments). See the explanation in Remark 4, after the proof of Theorem 6. (For capacity, and potential theory, cf. the books [HK], [A79], [A91], [Ra] and [AG].)

Now we give a non-trivial example of (real) analytic families of surjective homomorphisms (*-homomorphisms) between Banach algebras (Banach *-algebras, with continuous involutions). Essentially the same example was provided independently by Globevnik [Glo3] and Leiterer [Le2].

Example 1. Let $D:=\{z \in \mathbb{C}:|z|<1\}$, and let $n \geq 1$ be an integer. Let $B$ be a Banach algebra (Banach *-algebra, with a continuous involution); here the existence of unit is not required. Let $A$ be the following Banach algebra (in general, also without unit) of analytic functions $D^{n} \rightarrow B$ :

$$
A:=\left\{f: D^{n} \rightarrow B: f=\sum_{0}^{\infty} a_{k_{1} \ldots k_{n}} z_{1}^{k_{1}} \ldots z_{n}^{k_{n}}, a_{k_{1} \ldots k_{n}} \in B,\|f\|:=\sum_{0}^{\infty}\left\|a_{k_{1} \ldots k_{n}}\right\|<\infty\right\} .
$$

That is, $A$ is the set of absolutely convergent power series, when considered as functions $\bar{D}^{n} \rightarrow B$, with the norm given in the last display formula.

If $B$ is a Banach *-algebra with continuous involution, we let

$$
f^{*}:=\sum_{0}^{\infty} a_{k_{1} \ldots k_{n}}^{*} z_{1}^{k_{1}} \ldots z_{n}^{k_{n}}
$$

We observe that the now defined involution on $A$ is continuous, as well. Indeed, let

$$
\forall b \in B \quad\left\|b^{*}\right\| \leq C \cdot\|b\|,
$$


for some $C \in(0, \infty)$, and let $C$ be the smallest number such that this inequality holds. Then, we have

$$
\left\|f^{*}\right\|=\sum_{0}^{\infty}\left\|a_{k_{1} \ldots k_{n}}^{*}\right\| \leq C \cdot \sum_{0}^{\infty}\left\|a_{k_{1} \ldots k_{n}}\right\|=C \cdot\|f\|
$$

and also for this last inequality the number $C$ is the smallest number such that this inequality holds (this being true already for the smaller set of all constant functions $f$ of norm 1). So, the involution on $A$ is also continuous (and the constant $C$ will not appear anymore in the sequel).

We define

$$
\pi\left(\lambda_{1}, \ldots, \lambda_{n}\right) f:=f\left(\lambda_{1}, \ldots, \lambda_{n}\right),
$$

for each $\lambda_{1}, \ldots, \lambda_{n} \in D$. This is a surjective homomorphism, and, in case of Banach ${ }^{*}$-algebras, for $\lambda_{1}, \ldots, \lambda_{n} \in(-1,1)$, this is even a ${ }^{*}$-homomorphism. We have the expansion

$$
\pi\left(\lambda_{1}, \ldots, \lambda_{n}\right) f=\sum_{0}^{\infty} a_{k_{1} \ldots k_{n}} \lambda_{1}^{k_{1}} \ldots \lambda_{n}^{k_{n}}=: \sum_{0}^{\infty} a_{k_{1} \ldots k_{n}}(f) \lambda_{1}^{k_{1}} \ldots \lambda_{n}^{k_{n}}
$$

Here the maps $f \mapsto a_{k_{1} \ldots k_{n}}(f)$ are linear operators of norm 1 , so the above series gives the power series expansion of $\pi\left(\lambda_{1}, \ldots, \lambda_{n}\right)$, for $\lambda_{1}, \ldots, \lambda_{n} \in D$, hence $\pi\left(\lambda_{1}, \ldots, \lambda_{n}\right)$ is, in fact, an analytic family on $D^{n}$. It is interesting to observe, that $\pi\left(\lambda_{1}, \ldots, \lambda_{n}\right)$, for each $\lambda_{1}, \ldots, \lambda_{n} \in D$, has norm 1 - hence their norm is constant — which norm is attained exactly for all constant functions $f\left(\lambda_{1}, \ldots \lambda_{n}\right)=a_{0 \ldots 0}(f)$. (In fact, for $\lambda_{1}, \ldots \lambda_{n} \in D$, we have $\left\|\pi\left(\lambda_{1}, \ldots, \lambda_{n}\right) f\right\|=\left\|\sum_{0}^{\infty} a_{k_{1} \ldots k_{n}}(f) \lambda_{1}^{k_{1}} \ldots \lambda_{n}^{k_{n}}\right\| \leq$ $\sum_{0}^{\infty}\left\|a_{k_{1} \ldots k_{n}}(f)\right\| \cdot\left|\lambda_{1}\right|^{k_{1}} \ldots\left|\lambda_{n}\right|^{k_{n}}<\sum_{0}^{\infty}\left\|a_{k_{1} \ldots k_{n}}(f)\right\|=\|f\|$, unless all $a_{k_{1} \ldots k_{n}}$, for which $k_{1}+\ldots+k_{n}>0$, vanish.) For $B=\mathbb{C}$, we obtain a non-trivial holomorphic family of multiplicative linear functionals, of constant norm 1.

Analytic families in Banach spaces, with constant norm (which do not exist in the scalar valued case, unless they are constant), had been studied, e.g., in [TW], [GV1], [GV2], [Glo1], [Glo2]. Now we see that their values may consist of very natural objects, like surjective homomorphisms (even multiplicative linear functionals), to which our results are applicable.

Remark 3. For Theorems 5 and 6 the spectral hypothesis at the single point 0 does not extend to any larger set, like it does for Theorems 3 and 4, cf. Remarks 2 and 4. If in Example 1 we let $B:=\mathbb{C}$ and $n:=1$, then for $\pi(\lambda) z(\cdot):=z(\lambda)$ we have, for $z(\lambda):=\lambda$ and $\lambda=0$, that the spectrum of $\pi(\lambda) z(\cdot)$ for $\lambda=0$ is $\{0\}$, but for any $\lambda \in D \backslash\{0\}$ (for Theorem 6 for any $\lambda \in(-1,1) \backslash\{0\}$ ), we have that the spectrum of $\pi(\lambda) z(\cdot)=z(\lambda)=\lambda \in \mathbb{C}$ is $\{\lambda\} \neq\{0\}$. See also the explanation in Remark 4, after the proof of Theorem 6 .

Observe that in Example 1 we could not control the spectra of the elements in the kernels of $\pi\left(\lambda_{1}, \ldots, \lambda_{n}\right)$. Next we show a modification of this example, where $\pi\left(\lambda_{1}, \ldots, \lambda_{n}\right)$ is not constant, but, for the spectra of the elements of $\operatorname{Ker} \pi\left(\lambda_{1}, \ldots\right.$, $\left.\lambda_{n}\right)$, we have the strongest "smallness" property from all our theorems: namely, that all these spectra are $\{0\}$. In this modification, we may even have 
commutative Banach algebras.

Example 2. Let us specify $B$ in the above example, as follows. As a Banach space, $B=C([0,1])$ (of course with complex valued functions). We define the *-operation by conjugation of the functions. Then $B$ will become a Banach algebra (without unit), if multiplication is defined as the convolution (and also a *-algebra, with an isometric involution). If $f \in B$, then for its $n$th convolution power $f^{(* n)}$ we have $\left\|f^{(* n)}\right\| \leq\|f\|^{n} / n$ !, hence the spectral radius of any element is 0 , and the spectrum of any element is $\{0\}$. This readily implies that $B$ has no unit. (In fact, we could take for $B$ any commutative radical Banach algebra, of course, without unit.) We define $A$, and $\pi\left(\lambda_{1}, \ldots, \lambda_{n}\right)$, as in Example 1.

Then, also for $A$, we have that any element has spectral radius 0 , and hence, has spectrum $\{0\}$. In fact, for monomials this is evident. Then, taking into account that, for commutative Banach algebras, the spectral radius is subadditive, we have that the spectrum of any polynomial is $\{0\}$, as well. Last, we write any element $f \in A$, i.e., an absolutely convergent power series on $\bar{D}^{n}$, with coefficients from $B$, as

$f=\sum_{0}^{\infty} a_{k_{1} \ldots k_{n}} z_{1}^{k_{1}} \ldots z_{n}^{k_{n}}=\sum_{\max k_{i} \leq N} a_{k_{1} \ldots k_{n}} z_{1}^{k_{1}} \ldots z_{n}^{k_{n}}+\sum_{\max k_{i}>N} a_{k_{1} \ldots k_{n}} z_{1}^{k_{1}} \ldots z_{n}^{k_{n}}$

Here, on the right hand side, the first summand has spectral radius 0 , and the second summand has spectral radius at most the sum of the respective norms, which is arbitrarily small, if $N$ is sufficiently large. Hence, the spectral radius of $f$ is 0 , as asserted. Then, of course, for any $\left(\lambda_{1}, \ldots, \lambda_{n}\right) \in D^{n}$, we have that the spectra of all elements of $\operatorname{Ker} \pi\left(\lambda_{1}, \ldots, \lambda_{n}\right)(\subset A)$ are $\{0\}$ as well. The only problem is that both $B$ and $A$ are not unital.

Therefore, let us consider their unitizations $B \oplus \mathbb{C}$, and $A \oplus \mathbb{C}$, with the $l^{1}$-sum norms, which are Banach algebra norms. Then, each $\pi\left(\lambda_{1}, \ldots, \lambda_{n}\right)$ has a unique extension $\tilde{\pi}\left(\lambda_{1}, \ldots, \lambda_{n}\right): B \oplus \mathbb{C} \rightarrow A \oplus \mathbb{C}$, that is unital: we have $\tilde{\pi}\left(\lambda_{1}, \ldots, \lambda_{n}\right)(f \oplus$ $c):=\pi\left(\lambda_{1}, \ldots, \lambda_{n}\right)(f) \oplus c$, and this extension is a surjective algebra homomorphism as well. Let $f \oplus c \in \operatorname{Ker} \tilde{\pi}\left(\lambda_{1}, \ldots, \lambda_{n}\right)$. Then $0 \oplus 0=\tilde{\pi}\left(\lambda_{1}, \ldots, \lambda_{n}\right)(f \oplus c)=$ $\pi\left(\lambda_{1}, \ldots, \lambda_{n}\right)(f) \oplus c$, hence $0=c$, and $f \oplus c=f \oplus 0$. Therefore the spectral radius of $f \oplus c$ is 0 , and $\sigma(f \oplus c)=\{0\}$.

If we take $n=1$ and $B:=\mathbb{C}$ (which is unital), then we have that $A$ from Example 1 is the Banach algebra of absolutely convergent power series on $\bar{D}$ (which is also unital, and is not of the above form $B \oplus \mathbb{C}$ ). Here we have the opposite situation: for $f \in A$ we have $\sigma(f)=f(\bar{D})$, that may disconnect $\mathbb{C}$, even if $f$ is in the kernel of $\pi(\lambda)$, i.e., if $f(\lambda)=0$, for some $\lambda \in D$. We give a concrete example. Let us consider the open $\varepsilon_{0}$-neighbourhood $C_{\varepsilon_{0}}$ of the curve $C:=[0,1] \cup\{z \in \mathbb{C}$ : $|z|=1, \operatorname{Im} z \geq 0\}$, where $\varepsilon_{0}>0$ is sufficiently small. By the Riemann mapping theorem there is a bijective analytic map $f: D \rightarrow C_{\varepsilon_{0}}$, that can be extended to a homeomorphism of $\bar{D}$ onto $\overline{C_{\varepsilon_{0}}}$. Then $f^{2}(D)$ will be a small neighbourhood of $C^{2}=[0,1] \cup\{z \in \mathbb{C}:|z|=1\}$ (in fact, non-constant analytic functions are open maps). Hence it contains 0 , and disconnects $\mathbb{C}$. The only problem is that here possibly the power series of $f$ is not absolutely convergent. Therefore, let us restrict $f$ to $(1-\varepsilon) D$, for $\varepsilon>0$ sufficiently small, whose power series expansion 
is absolutely convergent on $(1-\varepsilon) \bar{D}$. Then still $f^{2}((1-\varepsilon) D)$ contains 0 , and $f^{2}((1-\varepsilon) \bar{D})$ disconnects $\mathbb{C}$. Thus, even the weakest spectral property from our theorems is not satisfied for this case.

Example 3. Unfortunately, in the algebra $A \oplus \mathbb{C}$ from the first part of Example 2 , each element has a one-point spectrum $(\sigma(f \oplus c)=\{c\})$, hence this algebra has no non-trivial idempotents. Next we show that all our hypotheses can be fulfilled simultaneously. Let $A_{1}$ be any Banach algebra (with continuous involution), with a non-central idempotent $e_{1}$ (e.g., all complex $n \times n$ matrices, for $n \geq 2$ ). Let $x_{1} \in A_{1}$, and $e_{1} x_{1} \neq x_{1} e_{1}$. Then, in $A_{1}$, we have a non-trivial analytic family of idempotents. Namely, for $\lambda \in \mathbb{C}$, close to 0 , we have $e_{1} e^{\lambda x_{1}} \neq e^{\lambda x_{1}} e_{1}$. Therefore, the element obtained from $e_{1}$ by a similarity via an exponential function, namely $e^{-\lambda x_{1}} e_{1} e^{\lambda x_{1}}$, is a non-trivial analytic family of idempotents, even defined for all $\lambda \in \mathbb{C}$. (More generally, compare also the theorem of Labrousse, [La], from the title of his paper.) Now we multiply both $B \oplus \mathbb{C}$, and $A \oplus \mathbb{C}$, by $A_{1}$ (taking on the Cartesian products the $l^{\infty}$-sum norms). Then extend $\tilde{\pi}\left(\lambda_{1}, \ldots, \lambda_{n}\right)$, as identity on $A_{1}$. Thus we obtain a non-trivial analytic family of surjective homomorphisms $(A \oplus$ $\mathbb{C}) \times A_{1} \rightarrow(B \oplus \mathbb{C}) \times A_{1}$. Then the elements of the kernels have a component in $A_{1}$ which is equal to 0 , hence the spectra of these elements are $\{0\}$. Simultaneously we have a non-trivial analytic family of idempotents in $(A \oplus \mathbb{C}) \times A_{1}$; their components in $(A \oplus \mathbb{C})$ are chosen as the identity, or zero, of $A \oplus \mathbb{C}$, and their components in $A_{1}$ are the above $e^{-\lambda x_{1}} e_{1} e^{\lambda x_{1}}$.

\section{Proofs of the THEOREMS}

Proof of Theorem 1. We follow [AMMZ, p. 25], which in turn followed the proof of $\pi E(A)=E(A / \operatorname{rad} A)$ for $\pi: A \rightarrow A / \operatorname{rad} A$ the canonical map, from [Ri, p. 58] and [Ka, p. 124].

The set of surjective bounded linear maps between Banach spaces is open in the corresponding operator space [Gle, Proposition 1.5], so we may suppose that $\pi(\lambda)$ is surjective for each $\lambda \in U$ ( $U$ can be decreased).

By a theorem of Harte, cf. [A91, p. 47, Theorem 3.3.8], for any $x \in A$ we have

$$
\sigma(\pi(0) x) \subset \cap\{\sigma(x+y): y \in \operatorname{Ker} \pi(0)\} \subset
$$

$\sigma(\pi(0) x) \cup(\cup\{G: G$ is a bounded connected component of $\mathbb{C} \backslash \sigma(\pi(0) x)\})$.

(The last set is also called the polynomially convex hull of $\sigma(\pi(0) x)$.)

Like in the proof of [AMMZ, Theorem 3.1], we may assume that $\sigma(q(0))=\{0,1\}$ (else $p \equiv 0$ or $p \equiv 1$ could be chosen). Applying Harte's theorem, we see that

$$
\cap\left\{\sigma(c): c \in \pi(0)^{-1} q(0)\right\}=\{0,1\} .
$$

Choose $c \in \pi(0)^{-1} q(0)$ such that $\sigma(c) \not \supset 1 / 2$.

By [Gle, Lemmas 1.7, 1.10], there is an open set $V \subset \mathbb{C}$, such that $0 \in V \subset U$, and an analytic map $a: V \rightarrow A$ such that $a(0)=c$ and

$$
\pi(\lambda) a(\lambda)=q(\lambda) \text { for each } \lambda \in V
$$


Hence $\sigma(a(0)) \not \supset 1 / 2$.

By upper semicontinuity of the spectrum [A79, p. 6, Théorème 1.1.3] or [A91, p. 50, Theorem 3.4.2], we may assume, by decreasing $V$, that

$$
\sigma(a(\lambda)) \not \ngtr 1 / 2, \text { for each } \lambda \in V \text {. }
$$

Then, by the spectral mapping theorem, we have

$$
\sigma\left(a(\lambda)^{2}-a(\lambda)\right) \not \ngtr-1 / 4, \text { for each } \lambda \in V \text {. }
$$

(For this observe that the only solution of the equation $\lambda^{2}-\lambda=-1 / 4$ in $\mathbb{C}$ is $\lambda=1 / 2$.)

Hereafter only small modifications are necessary compared to $[\mathrm{Ri}]$ and $[\mathrm{Ka}]$. We have

$$
-a(\lambda)^{2}+a(\lambda)=: r(\lambda) \in \operatorname{Ker} \pi(\lambda),
$$

with $r: V \rightarrow A$ analytic, such that $\sigma(r(\lambda)) \not \supset 1 / 4$, for each $\lambda \in V$. We want to obtain a solution $z(\lambda) \in \operatorname{Ker} \pi(\lambda)$, with $z: V \rightarrow A$ analytic, of the equation

$$
(a(\lambda)+z(\lambda))^{2}=a(\lambda)+z(\lambda)
$$

that commutes with $a(\lambda)$.

Due to this commutativity, (1) reduces to

$$
z(\lambda)^{2}+(2 a(\lambda)-1) z(\lambda)-r(\lambda)=0
$$

Since $1 / 2 \notin \sigma(a(\lambda))$, for each $\lambda \in V$, we may consider

$$
x(\lambda):=z(\lambda)(2 a(\lambda)-1)^{-1},
$$

which is analytic on $V$. Observing that $(2 a(\lambda)-1)^{2}=1-4 r(\lambda)$, we rewrite (2) as an equation for $x(\lambda)$, obtaining

$$
x(\lambda)^{2}+x(\lambda)-r(\lambda)(1-4 r(\lambda))^{-1}=0
$$

(recall that $\sigma(r(\lambda)) \not \supset 1 / 4$, for each $\lambda \in V)$. We write

$$
-r(\lambda)(1-4 r(\lambda))^{-1}=: r_{0}(\lambda) \in \operatorname{Ker} \pi(\lambda),
$$

where $r_{0}: V \rightarrow A$ is analytic. Observing that the map

$$
\mathbb{C} \backslash\{1 / 4\} \ni \lambda \mapsto-\lambda(1-4 \lambda)^{-1}
$$

does not contain $1 / 4$ in its image, the spectral mapping theorem gives

$$
\sigma\left(r_{0}(\lambda)\right) \not \supset 1 / 4, \text { for each } \lambda \in V \text {. }
$$


We rewrite (4) as

$$
x(\lambda)^{2}+x(\lambda)+r_{0}(\lambda)=0 .
$$

A formal solution of (5) would be

$$
x(\lambda)=-\frac{1}{2} \pm \frac{1}{2} \sqrt{1-4 r_{0}(\lambda)} .
$$

Observe that $\sigma\left(1-4 r_{0}(0)\right) \not \nexists 0$. Moreover, by the hypothesis of the theorem, $\sigma\left(1-4 r_{0}(0)\right)$ does not disconnect $\mathbb{C}$, since $r_{0}(0) \in \operatorname{Ker} \pi(0)$. Therefore there exists a simple polygonal arc $P$ connecting 0 with infinity, ending with a half-line, and avoiding $\sigma\left(1-4 r_{0}(0)\right)$. Hence, the distance of $P$ and $\sigma\left(1-4 r_{0}(0)\right)$ is positive. We write $\varepsilon \in(0, \infty)$ for the third of this distance.

By upper semicontinuity of the spectrum [A79, p. 6, Théorème 1.1.3] or [A91, p. 9, Theorem 3.4.2], for all $\lambda$ in some open set $V^{\prime}$, containing 0 , and contained in $V$, we have that

$$
\left\{\begin{array}{c}
\sigma\left(1-4 r_{0}(\lambda)\right) \text { lies in the closed } \varepsilon \text {-neighbourhood of } \sigma\left(1-4 r_{0}(0)\right), \\
\text { thus, in particular, it avoids the closed } \varepsilon \text {-neighbourhood of } P .
\end{array}\right.
$$

Denoting by $\rho(\cdot)$ the spectral radius, we have, by (7), for all $\lambda \in V^{\prime}$, that

$$
\rho\left(1-4 r_{0}(\lambda)\right) \leq \rho\left(1-4 r_{0}(0)\right)+\varepsilon .
$$

We may assume that the above open set $V^{\prime}$ equals $V$ ( $V$ may be decreased).

The function $\lambda \mapsto \lambda^{1 / 2}$ has two analytic branches on $\mathbb{C} \backslash P$. Therefore $\sqrt{1-4 r_{0}(\lambda)}$ can be defined by holomorphic calculus as

$$
\frac{1}{2 \pi i} \int_{\Gamma} z^{1 / 2}\left[z-\left(1-4 r_{0}(\lambda)\right)\right]^{-1} d z
$$

Here $z^{1 / 2}$ is any of the above two analytic branches. Moreover, $\Gamma \subset \mathbb{C} \backslash P$ is a (closed) Jordan polygon, which is the union of two closed arcs $\Gamma_{1}$ and $\Gamma_{2}$, with disjoint relative interiors. Here $\Gamma_{1}$ lies in the closed $\varepsilon$-neighbourhood of the simple polygonal $\operatorname{arc} P$, with both its endpoints having a distance at least $\rho\left(1-4 r_{0}(0)\right)+$ $2 \varepsilon$ from 0 , and $\Gamma_{2}$, having the same endpoints, has a distance from 0 at least $\rho\left(1-4 r_{0}(0)\right)+2 \varepsilon$. (So, $\Gamma_{1}$ has points close to 0 , as well as points far from 0 , but, anyway, it lies close to $P$. At the same time, $\Gamma_{2}$ has only points that are far from 0 . A proper choice for $\Gamma$, for the case when $P$ is the non-negative real axis, is the following. $\Gamma_{1}$ is the polygonal arc $(1 / \varepsilon,-\varepsilon)(0,-\varepsilon)(-\varepsilon, 0)(0, \varepsilon)(1 / \varepsilon, \varepsilon)$ and $\Gamma_{2}$ is the polygonal arc $(1 / \varepsilon, \varepsilon)(1 / \varepsilon, 1 / \varepsilon)(-1 / \varepsilon, 1 / \varepsilon)(-1 / \varepsilon,-1 / \varepsilon)(1 / \varepsilon,-1 / \varepsilon)(1 / \varepsilon,-\varepsilon)$. Cf. Fig. 1 in separate file, $\Gamma_{1}$ in continuous lines, $\Gamma_{2}$ in broken lines.) By (7), (8) and the construction of $\Gamma$, we have that

$$
\Gamma \text { encloses } \sigma\left(1-4 r_{0}(\lambda)\right) \text {, for all } \lambda \in V \text {. }
$$

Therefore, the definition given in formula (9) is correct. Then the function 
$\sqrt{1-4 r_{0}(\lambda)}$, defined by (9), is analytic on $V$, hence, by (6), $x: V \rightarrow A$ is analytic as well, and solves equation (5). Since $r_{0}(\lambda)$ is a rational function of $a(\lambda)$, they commute, hence, by (6) and (9), $x(\lambda)$ and $a(\lambda)$ commute. Next, by (3), $z(\lambda)=(2 a(\lambda)-1) x(\lambda)$ and $a(\lambda)$ commute as well, as we wanted to show. Since (5) is satisfied, each of (4), (2) and (1) is satisfied, as well. Lastly,

$\pi(\lambda)(a(\lambda)+z(\lambda))=\pi(\lambda)(a(\lambda)+(2 a(\lambda)-1) x(\lambda))=q(\lambda)+(2 q(\lambda)-1) \pi(\lambda)(x(\lambda))$

and, by (6) and (9), with $\Gamma$ satisfying (10), we have

$$
\begin{aligned}
\pi(\lambda)(x(\lambda)) & =-\frac{1}{2} \pm \frac{1}{2} \cdot \frac{1}{2 \pi i} \int_{\Gamma} z^{1 / 2}\left[z-\pi(\lambda)\left(1-4 r_{0}(\lambda)\right)\right]^{-1} d z \\
& =-\frac{1}{2} \pm \frac{1}{2} \cdot \frac{1}{2 \pi i} \int_{\Gamma} z^{1 / 2}(z-1)^{-1} d z
\end{aligned}
$$

(since $\left.\pi(\lambda) r_{0}(\lambda)=0\right)$. Since $\Gamma$, by $(10)$, encloses $\sigma\left(1-4 r_{0}(\lambda)\right)$, for each $\lambda \in V$, therefore it encloses also its subset $\sigma\left[\pi(\lambda)\left(1-4 r_{0}(\lambda)\right)\right]=\{1\}$. Then

$$
\frac{1}{2 \pi i} \int_{\Gamma} z^{1 / 2}(z-1)^{-1} d z
$$

is already independent of $\lambda$, depends only on $\Gamma$ and the chosen branch of $\lambda \mapsto \lambda^{1 / 2}$, and has a value 1 or -1 . Therefore, by (11),

$$
\pi(\lambda)(x(\lambda))=-\frac{1}{2} \pm \frac{1}{2}( \pm 1)
$$

hence, with proper choice of the first \pm sign, we have $\pi(\lambda)(x(\lambda))=0$. Thus, by (3), we have

$$
\pi(\lambda)(z(\lambda))=0
$$

therefore

$$
\pi(\lambda)(a(\lambda)+z(\lambda))=q(\lambda)
$$

while, by (1), that we already know to hold, $a(\lambda)+z(\lambda)$ is idempotent, moreover $a(\lambda)+z(\lambda)$ is analytic on $V$.

Before the proof of Theorem 2 we recall some elementary concepts from topology. Total disconnectedness of a topological space was recalled in the first paragraph of Section 2. A topological space is 0-dimensional if it is non-empty, and has an open base consisting of open-and-closed sets. In particular, the totally disconnected spectra, mentioned in the first paragraph of Section 2, are 0-dimensional (cf. P1 below). For these spaces the following two properties are well known.

P1. Total disconnectedness and 0-dimensionality are equivalent for non-empty compact Hausdorff spaces [E, p. 362, Theorem 6.2.10, and p. 388, Theorem 7.1.12], and, for the non-empty compact metric case, [Ku, p. 189, Section 47, IX, 2nd paragraph] (but they are not equivalent for general spaces, cf. [Ku, p. 152, Section 46, VI, Remark (i)]). (Observe that both [Ku, p. 151, Section 46, VI, Definition] 
and [E, pp. 356, 360, 369] call total disconnectedness "hereditary disconnectedness", which is a rather obsolete terminology. What [Ku, p. 151, Section 46, VI, Definition] calls total disconnectedness, is another property, not used in our paper.) We note that we use these properties only for spectra of elements in Banach algebras, which are non-empty and compact.

P2. The complement of a 0-dimensional subset of the plane is connected $[\mathrm{Ku}$, p. 188, Section 47, VIII, Theorem 1, and p. 466, Section 59, II, Theorem 1].

Proof of Theorem 2. Like in the proof of Theorem 1, we may suppose that $\sigma(q(0))=$ $\{0,1\}$. By analyticity, both $\pi$ and $q$ extend to analytic functions $\pi: U \rightarrow \mathcal{B}(A, B)$ and $q: U \rightarrow E(A)$ for some open set $U$ being the union of small discs about each point of $G$, with $G \subset U \subset G+i \mathbb{R} \subset \mathbb{C}$, hence with $U \cap \mathbb{R}=G$, and with each connected component of $U$ intersecting $G$. We have that $\pi(\lambda)$ is a homomorphism $A \rightarrow B$. Both the homomorphism property of $\pi(\lambda)$, and the idempotency of $q(\lambda)$ are consequences of the identity theorem for analytic functions. Like in the proof of Theorem 1, we may suppose that $\pi(\lambda)$ is surjective for each $\lambda \in U$.

As in the proof of Theorem 1 , there exists an open set $V \subset \mathbb{C}$ such that $0 \in V \subset$ $U$, and an analytic map $a: V \rightarrow A$, such that

$$
\pi(\lambda) a(\lambda)=q(\lambda) \text { for each } \lambda \in V \text {. }
$$

Let $\lambda \in V \cap \mathbb{R}$. Since $\pi(\lambda)$ is a *-homomorphism, therefore

$$
\pi(\lambda)\left(a(\lambda)^{*}\right)=(\pi(\lambda) a(\lambda))^{*}=q(\lambda)^{*}=q(\lambda)=\pi(\lambda) a(\lambda) .
$$

This implies

$$
q(\lambda)=\pi(\lambda) \frac{a(\lambda)+a(\lambda)^{*}}{2} \text { for each } \lambda \in V \cap \mathbb{R} .
$$

Here $a(\lambda)$ is analytic, $a(\lambda)^{*}$ is conjugate analytic, so their restrictions to $V \cap \mathbb{R}$ are real analytic. (Observe, that in the interior of a circle of convergence, we have that

$$
a(\lambda)=\sum_{0}^{\infty} a_{n}\left(\lambda-\lambda_{0}\right)^{n} .
$$

By continuity of the involution on $A$, this implies that

$$
a(\lambda)^{*}=\sum_{0}^{\infty} a_{n}^{*}\left(\bar{\lambda}-\bar{\lambda}_{0}\right)^{n} .
$$

So, in fact, $a(\lambda)^{*}$ is conjugate analytic on each open set of $\mathbb{C}$, where $a(\lambda)$ is analytic.)

Thus we have a real analytic, self-adjoint valued function

$$
a_{0}(\lambda):=\frac{a(\lambda)+a(\lambda)^{*}}{2}
$$

on $V \cap \mathbb{R}$, lifting $q(\lambda)$, for each $\lambda \in V \cap \mathbb{R}$, by (12). This function $a_{0}(\lambda)$ extends 
by analyticity to an analytic function $a_{0}(\lambda)$ on a neighbourhood of $V \cap \mathbb{R}$, contained

in $(V \cap \mathbb{R})+i \mathbb{R} \subset \mathbb{C}$. We may suppose that this neighbourhood is $V$ ( $V$ may be decreased). By analyticity of $\pi(\lambda), q(\lambda)$ and $a_{0}(\lambda)$, and the identity theorem for analytic functions, we have, by (12) and (13), for each $\lambda \in V$, that

$$
\pi(\lambda) a_{0}(\lambda)=q(\lambda) .
$$

This means that we may assume that our original lifting $a(\lambda)$ is self-adjoint valued on $V \cap \mathbb{R}$. Then, for each $\lambda \in V \cap \mathbb{R}$, we have

$$
\sigma(a(\lambda))=\overline{\sigma(a(\lambda))} .
$$

(Observe that in general we do not have that the spectrum of a self-adjoint element is real: in the Banach algebra of $\mathbb{C}$-valued absolutely convergent power series on $\bar{D}$, with norm $\left\|\sum_{n=0}^{\infty} c_{n} z^{n}\right\|:=\sum_{n=0}^{\infty}\left|c_{n}\right|$, and with involution given by coefficientwise conjugation, the self-adjoint element $f(z) \equiv z$ has spectrum $\bar{D}$.)

Then, like in the proof of [AMMZ, Theorem 3.1], we construct the Riesz idempotent $p(\lambda) \in A$, and elements $a_{0}(\lambda), a_{1}(\lambda) \in A$. These functions are analytic on a neighbourhood of 0 ; we may suppose that this neighbourhood is $V$ ( $V$ may be decreased). Namely,

$$
\begin{aligned}
p(\lambda) & :=\frac{1}{2 \pi i} \int_{\Gamma_{1}}(z-a(\lambda))^{-1} d z \\
a_{0}(\lambda) & :=\frac{1}{2 \pi i} \int_{\Gamma_{0}}(1-z)^{-1}(z-a(\lambda))^{-1} d z \\
a_{1}(\lambda) & :=\frac{1}{2 \pi i} \int_{\Gamma_{1}} z^{-1}(z-a(\lambda))^{-1} d z
\end{aligned}
$$

where $\Gamma_{0}$ and $\Gamma_{1}$ are Jordan polygons, their interior domains $\operatorname{Int} \Gamma_{i}$ being disjoint, and together covering $\sigma(a(0))$, with $0 \in \operatorname{Int} \Gamma_{0}$ and $1 \in \operatorname{Int} \Gamma_{1}$. These exist, as asserted in [AMMZ, p. 25], as soon as we know that $\sigma(a(0))$ is totally disconnected - that will be proved in the next paragraph - due to the following facts.

1) The set $\sigma(a(0))$ is the union of two of its relatively open-and-closed subsets, one containing 0 , the other one containing 1 , which follows from the fact that total disconnectedness of $\sigma(a(0))$ implies that $\sigma(a(0))$ is 0-dimensional, cf. P1, and hence $\mathbb{C} \backslash \sigma(a(0))$ is connected, cf. P2.

2) Connected open subsets of the plane are connected via polygonal arcs $[\mathrm{Ku}, \mathrm{p}$. 461, Section 59, I, Theorem 1].

Now we show that $\sigma(a(0))$ is totally disconnected (as mentioned in [AMMZ, p. $25]$ without proof). In fact, we have $\pi(\lambda)\left(a(\lambda)^{2}-a(\lambda)\right)=q(\lambda)^{2}-q(\lambda)=0$, for each $\lambda \in V$. Hence, in particular,

$$
a(0)^{2}-a(0) \in \operatorname{Ker} \pi(0) .
$$

By our spectral hypothesis, $\sigma\left(a(0)^{2}-a(0)\right) \subset \mathbb{C}$ is totally disconnected. 
If $\sigma(a(0)) \subset \mathbb{C}$ were not totally disconnected, it would contain, by definition (recalled in the first paragraph of Section 2), a connected subset

$$
S \subset \sigma(a(0))
$$

consisting of more than one points. Then the image of $S$, under the continuous map $f: \mathbb{C} \rightarrow \mathbb{C}$, defined by $f(z):=z^{2}-z$, i.e., the set $f(S)$, is connected as well. Clearly, the connected set $S \subset \mathbb{C}$ cannot consist of exactly two points. Therefore, let $s_{1}, s_{2}, s_{3} \in S$ be distinct. Now observe that the inverse image of any $w \in \mathbb{C}$ under $f$ consists of at most two points. Therefore, the subset $\left\{f\left(s_{1}\right), f\left(s_{2}\right), f\left(s_{3}\right)\right\}$ of $f(S)$ consists of at least two points. Now, also using the spectral mapping theorem, we have

$$
\left\{f\left(s_{1}\right), f\left(s_{2}\right), f\left(s_{3}\right)\right\} \subset f(S) \subset f(\sigma(a(0)))=\sigma(f(a(0)))=\sigma\left(a(0)^{2}-a(0)\right) .
$$

Hence, $f(S)$ is a connected subset of $\sigma\left(a(0)^{2}-a(0)\right)$, consisting of more than one points, while $\sigma\left(a(0)^{2}-a(0)\right)$ is totally disconnected, that is a contradiction. Hence, $\sigma(a(0))$ is totally disconnected, as well, as promised to be shown at the beginning of this paragraph.

By upper semicontinuity of the spectrum $[\mathrm{A} 79, \mathrm{p}$. 6, Théorème 1.1.3] or [A91, p. 50, Theorem 3.4.2], we may suppose that this decomposition of the spectrum by the Jordan polygons $\Gamma_{0}$ and $\Gamma_{1}$ (i.e., being covered by $\left.\left(\operatorname{Int} \Gamma_{0}\right) \cup\left(\operatorname{Int} \Gamma_{1}\right)\right)$ holds for all $a(\lambda)$, where $\lambda \in V$ ( $V$ may be decreased $)$. Like in the proof of [AMMZ, Theorem 3.1 , we obtain

$$
a(\lambda)-p(\lambda)=\left(a(\lambda)^{2}-a(\lambda)\right)\left(a_{1}(\lambda)-a_{0}(\lambda)\right) \in \operatorname{Ker} \pi(\lambda)
$$

(For the reader's convenience, we sketch its proof. We have $p(\lambda)=a(\lambda) a_{1}(\lambda)$, and $1-p(\lambda)=(1-a(\lambda)) a_{0}(\lambda)$, hence $a(\lambda)-p(\lambda)=a(\lambda)(1-p(\lambda))-(1-a(\lambda)) p(\lambda)=$ $a(\lambda)(1-a(\lambda)) a_{0}(\lambda)-(1-a(\lambda)) a(\lambda) a_{1}(\lambda)$.) Therefore

$$
\pi(\lambda) p(\lambda)=\pi(\lambda) a(\lambda)=q(\lambda) \text { for each } \lambda \in V
$$

It remains to ensure that $p(\lambda)=p(\lambda)^{*}$ for $\lambda \in H:=V \cap \mathbb{R}$. However, by $\sigma(a(0))=\overline{\sigma(a(0))}$, we can choose $\Gamma_{1}$ symmetric with respect to the real axis, which yields $p(\lambda)=p(\lambda)^{*}$, for each $\lambda \in H$.

Proof of Theorem 3. The proof is analogous to those of [Ka, pp. 125-126, Theorem 31, Corollary]. For convenience, we use the notation of [Ka].

We only have to prove the statement corresponding to [Ka, p. 125, Theorem 31], since [Ka, p. 126, Corollary] is an easy consequence, whose proof can be taken over without modification (cf. the last paragraph of this proof).

Thus we only have to prove the following statement. Let $u, v: U \rightarrow E(B)$ be analytic, with $u(\lambda)$ and $v(\lambda)$ orthogonal, for each $\lambda \in U$. Let $e: U \rightarrow E(A)$ 
be analytic, with $\pi(\lambda) e(\lambda)=u(\lambda)$ for each $\lambda \in U$. Then there exists $f: U \rightarrow$ $E(A)$ analytic, such that

$$
\left\{\begin{array}{c}
e(\lambda) \text { and } f(\lambda)=f(\lambda)^{2} \text { are orthogonal for each } \lambda \in U, \\
\text { and } \pi(\lambda) f(\lambda)=v(\lambda) \text { for each } \lambda \in U .
\end{array}\right.
$$

By [Le1, Theorem 5.1] there exists $b: U \rightarrow A$ analytic, such that

$$
\pi(\lambda) b(\lambda)=v(\lambda) \text { for each } \lambda \in U .
$$

Then, following $[\mathrm{Ka}]$, we define

$$
a(\lambda):=(1-e(\lambda)) b(\lambda)(1-e(\lambda)) .
$$

Then

$$
\pi(\lambda) a(\lambda)=v(\lambda),
$$

and $e(\lambda) a(\lambda)=a(\lambda) e(\lambda)=0$, and

$$
z(\lambda):=a(\lambda)^{2}-a(\lambda) \in \operatorname{Ker} \pi(\lambda),
$$

with $e(\lambda) z(\lambda)=z(\lambda) e(\lambda)=0$.

Then, by hypothesis,

$$
\sigma(z(\lambda))=\{0\},
$$

and therefore $(2 a(\lambda)-1)^{2}=1+4 z(\lambda)$ is invertible. Also, by the spectral mapping theorem, we have by (15) that

$$
\sigma(a(\lambda)) \subset\{0,1\} .
$$

Then, following $[\mathrm{Ka}]$, we solve the equation

$$
w(\lambda)^{2}+w(\lambda)+z(\lambda)(2 a(\lambda)-1)^{-2}=0,
$$

where $w: U \rightarrow A$ is a function. One of the formal solutions of (17) is

$$
w(\lambda)=-\frac{1}{2}+\frac{1}{2} \sqrt{1-4 z(\lambda)(2 a(\lambda)-1)^{-2}} .
$$

Here

$$
4 z(\lambda)(2 a(\lambda)-1)^{-2} \in \operatorname{Ker} \pi(\lambda),
$$

hence

$$
\sigma\left(4 z(\lambda)(2 a(\lambda)-1)^{-2}\right)=\{0\} .
$$


Therefore, by functional calculus, $w(\lambda)$ can be defined as

$$
w(\lambda):=-\frac{1}{2}+\frac{1}{2} \frac{1}{2 \pi i} \int_{\Gamma}(1-\zeta)^{1 / 2}\left[\zeta-4 z(\lambda)(2 a(\lambda)-1)^{-2}\right]^{-1} d \zeta
$$

where $\Gamma$ is a small circle with centre at 0 , and we take that branch of $(1-\zeta)^{1 / 2}$, that takes some positive values on $\Gamma$. Then $w: U \rightarrow A$ is analytic, and solves equation (17), and $w(\lambda)$ commutes with $a(\lambda)$ and $e(\lambda)$. Moreover, $w(\lambda) \in \operatorname{Ker} \pi(\lambda)$, since

$$
\begin{aligned}
\pi(\lambda) w(\lambda) & =-\frac{1}{2}+\frac{1}{2} \cdot \frac{1}{2 \pi i} \int_{\Gamma}(1-\zeta)^{1 / 2}\left[\zeta-\pi(\lambda)\left(4 z(\lambda)(2 a(\lambda)-1)^{-2}\right)\right]^{-1} d \zeta \\
& =-\frac{1}{2}+\frac{1}{2} \cdot \frac{1}{2 \pi i} \int_{\Gamma}(1-\zeta)^{1 / 2}(\zeta-0)^{-1} d \zeta=-\frac{1}{2}+\frac{1}{2} \cdot 1=0 .
\end{aligned}
$$

Let

$$
x(\lambda):=(1-e(\lambda)) w(\lambda)=w(\lambda)(1-e(\lambda)) \in \operatorname{Ker} \pi(\lambda),
$$

with $x: U \rightarrow A$ analytic. Then $e(\lambda) x(\lambda)=x(\lambda) e(\lambda)=0$. Let $r: U \rightarrow A$ be the analytic function, defined by

$$
r(\lambda):=x(\lambda)(2 a(\lambda)-1)=(2 a(\lambda)-1) x(\lambda) \in \operatorname{Ker} \pi(\lambda) .
$$

Note that $r(\lambda)$ commutes with $a(\lambda)$. (Actually, all elements $a(\lambda), e(\lambda), z(\lambda), w(\lambda)$, $x(\lambda), r(\lambda)$ lie in a commutative subalgebra of $A$, depending on $\lambda$, as can be seen from their definitions, step by step. Despite the delicate fact that $a(\lambda)$ 's, for different $\lambda$ 's, may not be commuting!)

Let $f: U \rightarrow A$ be the analytic function, defined by

$$
f(\lambda):=a(\lambda)+r(\lambda) .
$$

Then, like in [Ka] (by essentially straightforward calculations, using commutativity of $a(\lambda), r(\lambda)$, and (17)), we obtain

$$
f(\lambda)^{2}=f(\lambda), \text { and } e(\lambda) f(\lambda)=f(\lambda) e(\lambda)=0 .
$$

Finally,

$$
\pi(\lambda) f(\lambda)=\pi(\lambda) a(\lambda)=v(\lambda) .
$$


This ends the proof of (14).

Now the proof of [Ka, p. 126, Corollary] finishes the proof of our theorem. (For the reader's convenience, we recall that, to define $p_{k}$ if $p_{1}, \ldots p_{k-1}$ already were defined, (14) was there applied for $u:=q_{1}+\cdots+q_{k-1}$, and $v:=q_{k}$, and $e:=$ $p_{1}+\cdots+p_{k-1}$; here, for $k=1$, an empty sum means 0 .)

Proof of Theorem 4. Again we proceed like [Ka, pp. 125-126, Theorem 31, Corollary]. Once more, we only have to prove the statement corresponding to [Ka, p. 125, Theorem 31], since [Ka, p. 126, Corollary] is an easy consequence, whose proof can be taken over without modification (cf. the last paragraph of this proof).

Thus we only have to prove the following statement. Let $u, v: G \rightarrow S(B)$ be real analytic, with $u(\lambda)$ and $v(\lambda)$ orthogonal, for each $\lambda \in G$. Let $e: G \rightarrow S(A)$ be real analytic, such that $\pi(\lambda) e(\lambda)=u(\lambda)$ for each $\lambda \in G$. Then there exists $f: G \rightarrow S(A)$ real analytic, such that

$$
\left\{\begin{array}{c}
e(\lambda) \text { and } f(\lambda)=f(\lambda)^{2}=f(\lambda)^{*} \text { are orthogonal for each } \lambda \in G, \\
\text { and } \pi(\lambda) f(\lambda)=v(\lambda) \text { for each } \lambda \in G .
\end{array}\right.
$$

Like in the proof of Theorem 2, by analyticity, $\pi, u, v$ and $e$ extend to analytic functions $\pi: U \rightarrow \mathcal{B}(A, B)$, and $u, v: U \rightarrow E(B)$, and $e: U \rightarrow E(A)$ for some open set $U \subset \mathbb{C}$ such that $G \subset U \subset G+i \mathbb{R} \subset \mathbb{C}$, thus $U \cap \mathbb{R}=G$, with each connected component of $U$ intersecting $G$, and with the following properties. We have that $\pi(\lambda)$, for each $\lambda \in U$, is a surjective homomorphism $A \rightarrow B$, and, for each $\lambda \in G$, each $x \in \operatorname{Ker} \pi(\lambda)$ satisfies $\sigma(x)=\{0\}$. Further, $u(\lambda)$ and $v(\lambda)$ are orthogonal for each $\lambda \in U$, and $\pi(\lambda) e(\lambda)=u(\lambda)$ for each $\lambda \in U$ (again, by using the identity theorem for analytic functions). Also at each further step, when the open set $U \subset \mathbb{C}$, containing $G$, is decreased, we always suppose that each connected component of $U$ intersects $G$.

Then we are in the situation of Theorem 3, except that we do not have $\sigma(x)=\{0\}$ for each $x \in \operatorname{Ker} \pi(\lambda)$, and for each $\lambda \in U$. (However, see Remark 4 after the proof of Theorem 6!) We have used this hypothesis, for $x=z(\lambda)$, i.e., $\sigma(z(\lambda))=\{0\}$, cf. (15), to prove that $\sigma(a(\lambda)) \subset\{0,1\}$ - and hence $(2 a(\lambda)-1)^{-2}$ exists and $\sigma\left(4 z(\lambda)(2 a(\lambda)-1)^{-2}\right)=\{0\}$, cf. (16), (18). By upper semicontinuity of the spectrum [A79, p. 6, Théorème 1.1.3] or [A91, p. 50, Theorem 3.4.2], and possibly decreasing $U$ at the respective steps of the proof, we may suppose the following three facts (23), (24), (25) (where always $\lambda \in U)$.

After possibly decreasing $U$, we may suppose, for some sufficiently small $\varepsilon>0$, that for all $\lambda \in U$, we have

$$
\sigma(z(\lambda)) \subset\{z \in \mathbb{C}:|z|<\varepsilon\}
$$

Namely, by upper semicontinuity of the spectrum, each point in $G$ has an open neighbourhood, contained in $U$, on which (23) holds. Then the new (decreased) $U$ will be the union of all these neighbourhoods. Therefore, by the spectral mapping theorem, we have, for each $\lambda \in U$, rather than (16), 


$$
\sigma(a(\lambda)) \subset\{z \in \mathbb{C}:|z|<1 / 3 \text { or }|1-z|<1 / 3\}
$$

Then (24) implies that $(2 a(\lambda)-1)^{-2}$ exists, for each $\lambda \in U$. Next, possibly decreasing $\varepsilon>0$ and $U$, similarly as above, we may suppose, for each $\lambda \in U$, rather than (18), that

$$
\sigma\left(4 z(\lambda)(2 a(\lambda)-1)^{-2}\right) \subset\{z \in \mathbb{C}:|z|<1 / 3\}
$$

Furthermore, we choose $\Gamma$ in $(19)$ as $\{z \in \mathbb{C}:|z|=1 / 2\}$.

Then the proof of (14) in the proof of Theorem 3 goes through. Therefore, there exists $f: U \rightarrow E(A)$ analytic, such that $e(\lambda)$ and $f(\lambda)$ are orthogonal, for each $\lambda \in U$, and $\pi(\lambda) f(\lambda)=v(\lambda)$, for each $\lambda \in U$. The only property that remains to be ensured is that $f(\lambda)$ is self-adjoint, for each $\lambda \in U \cap \mathbb{R}=G$. Of course, the proof of Theorem 3 does not give this. We will have to go over the proof of Theorem 3 , and step by step we will have to do the respective modifications, that finally will ensure that $f(\lambda)$ is self-adjoint, for each $\lambda \in G$. Like in the proof of Theorem 3 , there exists $b: U \rightarrow A$ analytic, such that $\pi(\lambda) b(\lambda)=v(\lambda)$ for each $\lambda \in U$. Like in the proof of Theorem 2, we even may assume (by changing $b(\lambda)$ ) that $b(\lambda)$ is self-adjoint, for each $\lambda \in U \cap \mathbb{R}=G$. (At the same time possibly $U$ has to be decreased, still each connected component of $U$ intersecting $G$.)

Then $a(\lambda)$ is self-adjoint for each $\lambda \in G$, as well as $z(\lambda)$. Then, like in (23), (24), (25), we may assume, after possibly decreasing $U$, for each $\lambda \in U$, the following facts - which are actually of the form (23), (24), (25), but now for the changed, already self-adjoint $b(\lambda)$, etc. For some sufficiently small $\varepsilon>0$, we have

$$
\sigma(z(\lambda)) \subset\{z \in \mathbb{C}:|z|<\varepsilon\}
$$

and hence $\sigma(a(\lambda)) \subset\{z \in \mathbb{C}:|z|<1 / 3$ or $|1-z|<1 / 3\}$ - and hence $(2 a(\lambda)-1)^{-2}$ exists - and $\sigma\left(4 z(\lambda)(2 a(\lambda)-1)^{-2}\right) \subset\{z \in \mathbb{C}:|z|<1 / 3\}$. Then, taking $\Gamma:=$ $\{z \in \mathbb{C}:|z|=1 / 2\}$ in (19), we have that $w(\lambda)$, defined by (19) and this $\Gamma$, is self-adjoint, for each $\lambda \in U \cap \mathbb{R}=G$. Observe that the product of two commuting self-adjoint elements is self-adjoint. Therefore, $x(\lambda)$ is self-adjoint, for each $\lambda \in G$, and then $r(\lambda)$ is self-adjoint, for each $\lambda \in G$, hence, finally, $f(\lambda)$ is self-adjoint, for each $\lambda \in G$, as was to be proven.

Now the analogue of the proof of [Ka, p. 126, Corollary] (cf. the last paragraph of the proof of Theorem 3) finishes the proof of our theorem.

Proof of Theorem 5. 
We follow the proofs of Theorems 3 and 4 .

As in the proof of Theorem 1, we may assume that $\pi(\lambda)$ is surjective for each $\lambda \in U$.

It suffices to prove the analogue of the statement in the third paragraph of the proof of Theorem 3, where however, rather than $f: U \rightarrow E(A)$, we have only $f: V \rightarrow E(A)$, for some open set $V \subset \mathbb{C}$, such that $0 \in V \subset U$, and (14) holds only for each $\lambda \in V$ (at both places).

As in the proof of Theorem 4, like in (23), (24), (25), we may assume, after possibly decreasing $V(\ni 0)$ at the respective steps, for each $\lambda \in V$, the following facts. For some sufficiently small $\varepsilon>0$, we have

$$
\sigma(z(\lambda)) \subset\{z \in \mathbb{C}:|z|<\varepsilon\}
$$

so that $\sigma(a(\lambda)) \subset\{z \in \mathbb{C}:|z|<1 / 3$ or $|1-z|<1 / 3\}$ - hence $(2 a(\lambda)-1)^{-2}$ exists - and $\sigma\left(4 z(\lambda)(2 a(\lambda)-1)^{-2}\right) \subset\{z \in \mathbb{C}:|z|<1 / 3\}$. Then $\Gamma$ can be chosen in (19) as $\{z \in \mathbb{C}:|z|=1 / 2\}$. With these changes, we arrive at formulas (20) and (21), however, only for $\lambda \in V$.

Last, the proof of [Ka, p. 126, Corollary] (cf. the last paragraph of the proof of Theorem 3) finishes the proof of our theorem. (Observe that if we would have had an infinite sequence $q_{1}, q_{2}, \ldots$ in the theorem, then the neighbourhoods of 0 in the inductive proof could shrink to 0 , but in finitely many steps this cannot occur.)

Proof of Theorem 6. We follow the proofs of Theorems 4 and 5. As in the proof of Theorem 1, we may assume that $\pi(\lambda)$ is surjective for each $\lambda \in G$. It suffices to prove the analogue of the statement in the second paragraph of the proof of Theorem 4, where however, rather than $f: G \rightarrow S(A)$, we have only $f: H \rightarrow S(A)$, for some open set $H \subset \mathbb{R}$, such that $0 \in H \subset G$, and (22) holds only for each $\lambda \in H$ (at both places).

As in the proofs of Theorems 4 and 5, like in (23), (24), (25), we may assume, after possibly decreasing $V(\ni 0)$ at the respective steps, for each $\lambda \in V$, the following facts. For some sufficiently small $\varepsilon>0$, we have

$$
\sigma(z(\lambda)) \subset\{z \in \mathbb{C}:|z|<\varepsilon\}
$$

and hence $\sigma(a(\lambda)) \subset\{z \in \mathbb{C}:|z|<1 / 3$ or $|1-z|<1 / 3\}$ - so that $(2 a(\lambda)-1)^{-2}$ exists - and $\sigma\left(4 z(\lambda)(2 a(\lambda)-1)^{-2}\right) \subset\{z \in \mathbb{C}:|z|<1 / 3\}$. Then $\Gamma$ can be chosen 
in (19) as $\{z \in \mathbb{C}:|z|=1 / 2\}$. With these changes, also changing $b(\lambda)$ to a self-adjoint element, for $\lambda \in H$, as in the proofs of Theorems 2 and 4, we arrive at (22), however, only for $\lambda \in H$ (at both places), where $H \subset \mathbb{R}$ is some open set, such that $0 \in H \subset G$.

Last, the analogue of the proof of [Ka, p. 126, Corollary] (cf. the last paragraph of the proof of Theorem 3) finishes the proof of our theorem.

Remark 4. Since, in the proof of Theorem 4, we have $\sigma(z(\lambda))=\{0\}$ for all $\lambda \in G$ (by $\pi(\lambda) z(\lambda)=0$ ), it follows that $\sigma(z(\lambda))=\{0\}$ for all $\lambda \in U$. Indeed, the logarithm of the spectral radius of $z(\lambda)$ is a subharmonic function on $U$, by Vesentini's theorem (see, e.g., [A79, p. 9, Théorème 1.2.1], [A91, p. 52, Theorem 3.4.7] or [Ra, p. 178, Theorem 6.4.2]). So, the above claim follows from [AG, p. 125, Corollary 5.1 .5 (i)] (the definition of a polar set mentioned there cf. with [HK, p. 212]), or, alternatively, from [A79, p. 11, Corollaire 1.2.1] with [A91, p. 180, Corollary A.1.27], [Ra, p. 42, Exercise 2.5.1], or their strengthenings [A91, p. 180, Theorem A.1.28], [Ra, p. 57, Exercise 3.2.1], and [HK, p. 225, Theorem 5.13] (the last one implies that the "non-degenerate straight line segments" in the last sentence of this paragraph can be replaced by compact sets of any positive Hausdorff dimension), applied to each connected component of $U$. The facts cited in the preceding sentence are particular cases of H. Cartan's theorem, stating, for a connected open set $\emptyset \neq U \subset \mathbb{C}$, that

1) if a subharmonic function $f: U \rightarrow[-\infty, \infty)$ is not identically $-\infty$ on $U$, then $f^{-1}(-\infty)$ is a $G_{\delta}$ set of capacity zero, and, conversely,

2) if $E \subset U$ is of zero capacity, then there exists a subharmonic function $f: U \rightarrow$ $[-\infty, \infty)$, not identically $-\infty$ on $U$, such that $E \subset f^{-1}(-\infty)$, cf., e.g., [HK, p. 274, Theorem 5.32], [A79, p. 173, Théorème 14], and [AZr, proof of Théorème 2.2]. (Concerning implication 1), see also [A91, p. 180, Theorem A.1.29] and [Ra, p. 65, Theorem 3.5.1], and its weaker form [Ra, p. 41, Corollary 2.5.3].) H. Cartan's theorem shows that the strongest spectral assumption on $\operatorname{Ker} \pi(\lambda)$, in Theorems 3 and 4 , can be postulated merely for $\lambda$ 's, e.g., on non-degenerate straight line segments in each connected component of $U$, or $G$.

Actually, both for Theorem 3 and Theorem 4, the strongest spectral assumption on $\operatorname{Ker} \pi(\lambda)$ can be postulated on any subsets $E$ of each connected component of $U$, or $G$, respectively, of positive outer capacity, but it is not sufficient to postulate them for subsets $E$ of zero capacity, if we only use H. Cartan's theorem for general subharmonic functions, as we have seen in this remark above, and especially in our earlier Remark 3. 
Cf. also Deny's theorem, [HK, p. 274] or [Ra, p. 65], which is a sharper form of H. Cartan's theorem above, and gives a complete characterization. Namely, if $E \subset \mathbb{C}$, and $\emptyset \neq U \subset \mathbb{C}$ is any connected open set containing $E$, then the following are equivalent:

1) there exists a subharmonic function $f: U \rightarrow[-\infty, \infty)$, not identically $-\infty$, such that $f^{-1}(-\infty)=E$;

2) $E$ is a $G_{\delta^{-}}$-set of capacity zero.

This is also a generalization of Evans' theorem [A91, p. 179, Theorem A.1.24] that is the particular case of Deny's theorem, for $E$ compact - used in the proofs of [AZr, Théorème 2.2] and [AMZ, p. 520, Lemma]. Let us also mention that we observed later that [AMZ, p. 520, Lemma] had been given already earlier, even in a more general form, as [AZr, Théorème 2.2], by the first mentioned author of [AMZ] and Zraïbi.

\section{Some PROBlems}

1) Does the conclusion of Theorem 2 hold, under the hypotheses of Theorem 2 , but replacing the hypothesis on spectra in Theorem 2 by the weaker one in Theorem 1 ?

2) An example of Gramsch [Gr] shows that for the canonical homomorphism $\pi: \mathcal{B}(H) \rightarrow \mathcal{C}(H)$ (where $H$ is a Hilbert space), and for an annulus $U$, it is not always possible to lift an analytic map $q: U \rightarrow E(\mathcal{C}(H))$ to the whole of $U$. Let us suppose in Theorem 1, additionally, that $U$ is simply connected. Furthermore, both in Theorem 1 and Theorem 2, let us additionally suppose that $\pi(\lambda)$ is surjective for each $\lambda \in U$, or for each $\lambda \in G$, and, in both cases, let us additionally suppose the respective spectral hypotheses for each element of $\operatorname{Ker} \pi(\lambda)$, for each $\lambda \in U$, or for each $\lambda \in G$, respectively. Can we then choose $V=U$, or $H=G$, respectively?

3) Suppose that all elements in $\operatorname{Ker} \pi(\lambda)$, for all $\lambda$ 's in a set which is large enough in some sense, satisfy one of the two topological spectral hypotheses, from Theorems 1 and 2. Does it follow, that all elements of all the kernels $\operatorname{Ker} \pi(\lambda)$, for $\lambda$ in our domain, satisfy the same spectral hypothesis? Observe that in Remark 4 we obtained satisfactory characterizations for the spectral hypothesis in Theorems 3 and 4 . The minimal such hypothesis would be for a set which is the union of sequences of $\lambda$ 's in each connected component of our domain, converging to points 
in the respective connected components of our domain. For the hypothesis of quasi-nilpotency, the sufficiency of this minimal hypothesis, for the canonical map to the Calkin algebra of a Banach space, was asked in [MZ]. More exactly, let $C(\lambda)$, for $\lambda \in D$ (= the open unit disc in $\mathbb{C}$ ), be an analytic family of compact operators in $\mathcal{B}(X)$, for a Banach space $X$. If $\sigma(C(\lambda))=\{0\}$ for a sequence of $\lambda$ 's in $D$, converging to 0 , does the same equality hold for all $\lambda \in D$ ? [MZ] proved that for analytic families $C(\lambda)$ of finite rank operators the answer is positive. This might also be related to the problems studied in [AZe].

Acknowledgements. We are grateful to Professors Béla Nagy (Szeged), Ivan Netuka, Szilárd Révész, and Yuri Tomilov for useful consultations on potential theory, especially for the references $[\mathrm{AG}]$ and [Ra] cited in Remark 4.

\section{REFERENCES}

[AG] D. H. Armitage, S. J. Gardiner, Classical Potential Theory, Springer Monographs in Math., Springer, London, 2001. MR 2001m:31001.

[A79] B. Aupetit, Propriétés Spectrales des Algèbres de Banach, Lecture Notes in Math. 735, Springer, Berlin-Heidelberg-New York, 1979. MR 81i:46055.

[A91] B. Aupetit, A Primer on Spectral Theory, Universitext, Springer, New York, 1991. MR 92c:46001.

[AMMZ] B. Aupetit, E. Makai, Jr., M. Mbekhta, J. Zemánek, The connected components of the idempotents in the Calkin algebra, and their liftings, in: Operator Theory and Banach Algebras, Conf. Proc., Rabat (Morocco), April 1999, Eds. M. Chidami, R. Curto, M. Mbekhta, F.-H. Vasilescu, J. Zemánek, Theta, Bucharest, 2003, 23-30. MR 2004g: 46062.

[AMZ] B. Aupetit, E. Makai, Jr., J. Zemánek, Strict convexity of the singular value sequences, Acta Sci. Math. (Szeged) 62 (1996), 517-521. MR 98h:47030.

[AZe] B. Aupetit, J. Zemánek, On zeros of analytic multivalued functions, Acta Sci. Math. (Szeged) 46 (1983), 311-316. MR 85f:30087.

[AZr] B. Aupetit, A. Zraïbi, Distribution des valeurs des fonctions analytiques multiformes, Studia Math. 79 (1984), 218-226. MR 86m:46046.

[C] J. W. Calkin, Two-sided ideals and congruences in the ring of bounded operators in Hilbert space, Ann. of Math. (2) 42 (1941), 839-873. MR 3,208c.

[DS] N. Dunford, J. T. Schwartz, Linear Operators, Part I: General Theory, Pure and Appl. Math. 7, Interscience, New York-London, 1958. MR 22\#8302.

[E] R. Engelking, General Topology, rev. and compl. ed., Sigma Ser. in Pure Math. 6, Heldermann, Berlin, 1989. MR 91c:54001.

[Gle] A. M. Gleason, Finitely generated ideals in Banach algebras, J. Math. Mech. 13 (1964), 125-132. MR 28\#2458.

[Glo1] J. Globevnik, On vector-valued analytic functions with constant norm, Studia Math. 53 (1975), 29-37. MR 52\#8916.

[Glo2] J. Globevnik, Norm-constant analytic functions and equivalent norms, Illinois J. Math. 20 (1976), 503-506. MR 56\#6354.

[Glo3] J. Globevnik, Letter (May 2011).

[GV1] J. Globevnik, I. Vidav, On operator-valued analytic functions with constant norm, J. Funct. Anal. 15 (1974), 394-403. MR 49\#11291.

[GV2] J. Globevnik, I. Vidav, On a class of vector-valued analytic functions, Ann. Polon. Math. 31 (1975), 73-81. MR 52\#3950.

[Gr] B. Gramsch, Lifting of idempotent operator functions, in: Banach Algebras '97, Proc. 13th Internat. Conf. on Banach Algebras (Blaubeuren, 1997), E. Albrecht and M. Mathieu, eds., de Gruyter, Berlin, 1998, 527-533. MR 99m:46119. 
[dlH] P. de la Harpe, Initiation à l'algèbre de Calkin, in: Algèbres d'Opérateurs (Sém., Les Plans-sur-Bex, 1978), Ed. P. de la Harpe, Lecture Notes in Math. 725, Springer, Berlin, 1979, 180-219. MR 81c:47045.

[HK] W. K. Hayman, P. B. Kennedy, Subharmonic Functions, Vol. I, London Math. Soc. Monographs 9, Academic Press, London etc., 1976. MR 57\#665.

[Ka] I. Kaplansky, Fields and Rings, 2nd ed., Chicago Lectures in Math., The University of Chicago Press, Chicago-London, 1972. MR 50\#2139.

$[\mathrm{Ku}] \quad$ K. Kuratowski, Topology II, Academic Press, New York-London; PWN Warsaw, 1968. MR 41\#4467.

[La] J.-Ph. Labrousse, The general local form of an analytic mapping into the set of idempotent elements of a Banach algebra, Proc. Amer. Math. Soc. 123 (1995), 3467-3471. MR 96a:46093.

[Le1] J. Leiterer, Banach coherent analytic Fréchet sheaves, Math. Nachr. 85 (1978), 91109. MR 80b:32026.

[Le2] J. Leiterer, Oral communication (May 2011).

[MZ] M. Mbekhta, J. Zemánek, Quasi-nilpotent and compact, in: Perspectives in Operator Theory, Banach Center Publ. 75, Proc. Workshop Operator Theory, Warsaw, 2004, Eds. W. Arendt, C. J. K. Batty, M. Mbekhta, Y. Tomilov, J. Zemánek, Polish Acad. Sci., Inst. Math., Warsaw, 2007, 318. MR 2008c:47001, MR2341355

[Ra] T. Ransford, Potential Theory in the Complex Plane, London Math. Soc. Student Texts 28, Cambridge University Press, Cambridge, 1995. MR 96e:31001.

[Ri] C. E. Rickart, General Theory of Banach Algebras, The University Series in Higher Math., van Nostrand, Princeton etc., 1960. MR 22\#5903.

[TW] E. Thorp, R. Whitley, The strong maximum modulus theorem for analytic functions into a Banach space, Proc. Amer. Math. Soc. 18 (1967), 640-646. MR 35\#5643.

Bernard Aupetit,

DÉPARTEMEnt De Mathématiques,

UNIVERSITÉ LAVAL,

QUÉBEC, G1K 7P4,

CANADA

ENDRE MAKAI, JR.,

Alfréd Rényi Mathematical institute,

Hungarian Academy of Sciences,

H-1364 Budapest, P.O. Box 127,

HUNGARY

E-mail address: makai.endre@renyi.mta.hu, http://www.renyi.mta.hu/ ${ }^{2}$ makai

Mostafa Mbekhta,

LABoratoire P. PAinlevé, UMR-CNRS 8524

U.F.R. De Mathématiques Pures et Appliquées, Bât. M2,

Université des Sciences et Technologies de Lille,

F-59655 Villeneuve D'AscQ,

FRANCE

E-mail address: mostafa.mbekhta@math.univ-lille1.fr

JAROSLAV ZEMÁNEK,

Institute of Mathematics, Polish Academy of Sciences, 00-956 Warsaw, P.O. Box 21,

POLAND

E-mail address: zemanek@impan.pl 